\title{
Validation of the QTNM Staging System for Cancer Specific Survival in Patients with Differentiated Thyroid Cancer
}

Short title: Validation of QTNM staging for thyroid cancer

Daniel Mankarios ${ }^{1}$, Peter Baade ${ }^{2}$, Pip Youl ${ }^{2}$, Robin H. Mortimer ${ }^{1,3}$, Adedayo A. Onitilo ${ }^{4,6}$, Anthony Russell ${ }^{1,5}$, Suhail A. R. Doi ${ }^{5,6}$

${ }^{1}$ School of Medicine, University of Queensland, Brisbane, Australia

${ }^{2}$ Cancer Council Queensland, Brisbane, Australia

${ }^{3}$ Royal Brisbane Hospital, Brisbane, Australia

${ }^{4}$ Department of Hematology/Oncology, Marshfield Clinic Weston Center, 3501 Cranberry Boulevard, Weston, WI 54476, USA

${ }^{5}$ Department of Endocrinology, Princess Alexandra Hospital, Brisbane, Australia

${ }^{6}$ Clinical Epidemiology Unit, School of Population Health, University of Queensland, Brisbane, Australia

Corresponding author's contact details:

s.doi@sph.uq.edu.au

Tel: +61 733655289

Keywords: Differentiated thyroid cancer, prognosis, QTNM, cancer staging, TNM 


\begin{abstract}
Background: An Australian state database was used to test the validity of the Quantitative Tumor/Node/Metastasis (QTNM) staging system for assessing prognosis of differentiated thyroid cancer (DTC) on the basis of four variables quantified at diagnosis (histopathology, age, node involvement and tumor size).
\end{abstract}

Methods: Using the Queensland Cancer Registry (QCR), we identified 788 cases of DTC diagnosed from 1982-2006 with complete staging information. Causes of death was ascertained by linking the QCR database with the Australian National Death Index. Subjects were staged according to AJCC TNM $7^{\text {th }}$ edition and QTNM, and cancer-specific survival (CSS) was calculated by the Kaplan-Meier method.

Results: Cancer-specific mortality was observed in $22(2.8 \%)$ patients, with ten-year CSS for the cohort of $97.0 \%$ at a median follow-up of 262.8 months. QTNM stage specific cancer survival at 10 years was 99.6\%, 97.0\% and 78.6\% for low, intermediate and high risk groups respectively. This was comparable to the original US dataset in which the QTNM was initially studied, and it fared better at discriminating survival than the standard TNM system where there was overlap in survival between stages.

Conclusion: The current study validates the QTNM system in an Australian cohort and shows at least equivalent discriminatory capacity to the current TNM staging system. The QTNM utilized prognostic variables of significance to produce an optimal three-stage stratification scheme. Given its advantage in clearly discriminating between prognostic groups, clinical relevance and simplicity of use, we recommend that TNM be replaced with QTNM for risk stratification for both recurrence and CSS. 


\section{Introduction}

Differentiated thyroid carcinoma (DTC) is both the most common endocrine malignancy as well as the ninth most prevalent cancer [1]. Its global incidence in the year 2008 is estimated at 211,748 which corresponds to a crude worldwide rate of 3.1 cases per 100,000 person years, making it the eighteenth most diagnosed cancer, with a female to male ratio of over 3 [2]. In Australia, thyroid cancer incidence rates have been steadily increasing from 2.7 cases per 100,000 people in 1982, to 9.1 cases per 100,000 in 2009 (Australian Institute of Health and Welfare (AIHW) 2012).

DTC has a favourable prognosis, with one large study estimating 10-year survival of between 76\%-93\%, depending on cell type [4]. As with most other cancers, multiple staging systems have been suggested in an attempt to predict the risk of recurrence and of cancer-related deaths. The American Joint Committee on Cancer (AJCC)/Union for International Cancer Control (UICC) Tumor/Node/Metastasis (TNM) staging system is the most widely used, and is considered the international gold standard and the staging system currently recommended by the American Thyroid Association [5]. The anatomic descriptors used in the AJCC/UICC classification produce a set of mutually exclusive and collectively exhaustive divisions, or "bins", into which patients are categorized into at the time of diagnosis [6]. This classification into bins has been thought to be useful for several reasons: (a) it provides information about prognosis which is derived from the mean survival of other patients in that bin [7], (b) it enables communication of accurate patient information between clinicians, and (c) it facilitates making treatment and follow-up decisions for the patient [5].

The problem, however, has been the proliferation of bins along with the recognition and incorporation of other non-anatomic variables of prognostic significance [8]. In addition, the TNM staging system has undergone multiple refinements since its inception with a consequent increase in the complexity of the binning system. As of its seventh edition, the AJCC TNM system has a total of 168 bins [9] $(7 \mathrm{~T} \times 3 \mathrm{~N} \times 2 \mathrm{M} \times 2$ age groups $\times 2$ histological subtypes $)$, up from 144 bins in edition six [10]. Bins then need to be transformed in to staging information through a "look-up" table which converts the 
various permutations of T, N, M and non-anatomic additions into one of 4 stages [7,10,5]. The collapse of such an extensive binning system into stages brings into question its practicality in the clinical setting. Indeed, the creation of stages from the bins may not be optimal because studies have found that survival may not be optimally discriminated between various stages so defined [4,11-14]. All this suggests that there may be no real benefit with the intermediary use of a binning system, but rather that prognostic risk factors should be directly connected to staging.

For these reasons, Onitilo et al [10] attempted to simplify TNM by developing a quantitative staging system, QTNM, which incorporates variables shown to be of prognostic significance directly into a prognostic stage. The system was developed using a Cox proportional hazards model on a cohort of 614 patients with DTC from the Marshfield Clinic in Wisconsin, United States, using disease-free survival (DFS) as the outcome. The model was then validated using a nested case control study from a Swedish cohort with DTC and it was demonstrated that this prognostic system had discriminative potential and allowed for accurate prediction of both CSS and DFS. Since there have been no subsequent validation studies in a prospective cohort, it was important to demonstrate its validity by applying it to a populationbased cohort of patients with DTC from Queensland, Australia while comparing it to the TNM staging system [15].

\section{Patients and methods}

Study Target Populations \& treatment protocol

Data from patients diagnosed with differentiated thyroid carcinoma (ICD-O3 C73) between January 1982 and December 2006 were electronically extracted from the Queensland Cancer Registry (QCR). The QCR is a population-based cancer registry, to which details of all cancers diagnosed in Queensland are legally required to be notified. Notifications of patients with cancer are received from all Queensland public and private hospitals and nursing homes, while public and private pathology laboratories are also required to provide copies of pathology reports for cancer specimens. Deaths among the thyroid cancer cohort are 
routinely identified by the QCR through matching patient information to the National Death Index (NDI) at the Australian Institute of Health and Welfare. People without a positive match with the NDI at $31^{\text {st }}$ December 2010 were assumed to be alive at that date. The QCR codes the cause of death using information from the ABS mortality file, pathology laboratories and other cancer-specific data sources, increasing the accuracy of the cause of death codes.

\section{Extraction of clinical data from pathology records}

Additional clinical information such as lesion diameter, evidence of metastasis, lymph node involvement, capsular invasion and extra-thyroid invasion are not routinely collected or reported for DTC by the QCR. As such this information was manually extracted from the pathology records held within the QCR. Information about these additional parameters was often missing from the pathology report, in particular a large proportion of subjects were missing tumour size, nodal status and location of involvement, or a combination of these. The data problem we faced with the pathology reporting in Queensland is not unique and a neighbouring registry (NSW Central Cancer Registry) reports a similar pattern of missing information on features important to the prognosis and treatment of thyroid cancer patients [16]. We undertook a comparison of extracted data and the original reports in 47 cases and noted that we could not reliably assume that absent values from the database were because they were negative. Cases with missing data were thus excluded from the analysis.

Approval

Approval to conduct this study was granted under Section 281 of the (Queensland) Public Health Act 2005 by Queensland Health, and ethical approval was obtained from the Human Research Ethics Committee at the University of Queensland. 


\section{Statistical Analysis}

The QTNM score is the sum of the following: Non-papillary thyroid cancer histopathology=1 point (Non-papillary differentiated thyroid cancers include follicular and Hurthle cell carcinoma but not poorly differentiated thyroid cancer as this staging system is only aimed at differentiated thyroid cancers), age $\geq 45$ years $=4$ points, regional lymph node metastasis $=4$ points, tumour $>4 \mathrm{~cm}$ or any tumour with extrathyroid extension=6 points. The QTNM score is then categorized into three risk stages based on scores 0-5; 6-10 and 11-15 representing low, intermediate and high risk categories respectively. Although this stratification method results in relatively fewer patients in the highest risk group, the original authors propose that it allows for ease of use with equivalent or better discrimination of this small subset of patients at high risk [10]. While this staging system was originally devised to provide prognostic information on recurrence-free survival, it was also shown to accurately discriminate CSS between different risk groups [10]. In this study, only CSS was investigated. The univariate relationships between CSS and the staging groups were done via the Kaplan-Meier method. The difference between two risk groups was compared with the Gehan-Wilcoxon test since this test gives more weight to deaths at early time points. Prognostic comparisons across stages were investigated using Cox regression analyses. No adjustments were made. None of the staging systems examined were associated with a gross violation of proportional hazards assumptions based on a visual inspection of the $-\ln (-\ln ($ survival $))$ vs. $\ln$ (survival time) plots. The discriminatory capacity of each prognostic staging system was tested using the Harrell's C statistic. [17] Harrell's C statistic estimates the proportion of correct predictions, i.e. the proportion of patients with a better prognostic stage who have a better survival and ranges between 0.5 (no discrimination) to 1 (perfect discrimination). Royston's D statistic [18] was also computed and estimates separation between independent survival distributions under the proportional hazards assumption. The higher the D statistic, the better is the discriminatory capacity and a difference in D of at least 0.1 indicates an important difference in separation between the relevant survival curves. Statistical analyses were performed using Stata version 11 computer software (StataCorp LP, College Station, TX, USA). 


\section{Comparisons to other staging systems}

There exist well over a dozen DTC staging systems which have been compared extensively in the literature. The majority of studies favour the use of AJCC TNM as the most accurate and reliable predictor of prognosis for DTC [19,11,20-23] and also specifically for follicular thyroid cancer (FTC) [24-26], followed by the Mayo Clinic's MACIS (metastases, age, completeness of resection, invasion, size) [27] for DTC $[28,29,26]$. We could not compare QTNM to MACIS due to the absence of details regarding completeness of resection, but our comparison to the TNM [30,31] system should be sufficient to demonstrate the reliability and accuracy of QTNM for two reasons. Firstly, QTNM is not a separate staging system, but rather a proposed change to the TNM system, and secondly this system uses similar prognostic variables. We used the $7^{\text {th }}$ edition of the AJCC TNM for staging, with one approximation: cases with absent location of nodal involvement were placed in stage III if over 45 years of age. The location of LN metatstases would categorize such patients as either stage III (N1a) or stage IV (N1b). We deliberately down-staged to improve TNM discrimination and avoid giving QTNM a false advantage.

\section{Results}

We retrieved data on 3,736 patients with DTC, of whom 2,948 had missing primary tumour size information ( 0 or absent) or absent nodal status and were therefore excluded as per the data validation discussed above. Of the patients that were excluded, 118 had died of thyroid cancer recurrence, and 326 died of other causes. The remaining 788 patients with complete staging information were used in the validation of QTNM. Mean age of subjects at diagnosis was 43.5 years (standard deviation 14.9), a total of 22 patients (2.8\%) died of thyroid cancer recurrence and 33 patients (3.5\%) died of other causes. Other relevant patient characteristics are shown in Table 1. We acknowledge that the distribution of subjects across the TNM stages is atypical, but upon investigation, no reasonable explanation could be found. 
Since this validation study is only examining the prognostic value of staging systems, this skewed distribution of subjects is insignificant as long as staging is accurately performed. We were however able to stage 2072 patients by TNM-7 criteria because tumor size and nodal status is not utilized under age 45 .

However, these patients could not be compared head to head with QTNM and were thus analysed separately.

Table 1. Characteristics of the population with DTC in terms of difference in survival between subgroups

\begin{tabular}{|c|c|c|c|}
\hline Patient Characteristics & $\mathbf{N}(\%)$ & $\begin{array}{l}\text { Events } \\
\text { observed }\end{array}$ & $\begin{array}{l}\text { CSS P } \\
\text { value }\end{array}$ \\
\hline Gender & & & 0.0323 \\
\hline Male & $215(27.3)$ & 10 & \\
\hline Female & $573(72.7)$ & 12 & \\
\hline Age & & & $<0.0001$ \\
\hline$<45$ & $455(57.5)$ & 4 & \\
\hline$\geq 45$ & $333(42.3)$ & 18 & \\
\hline Histopathology & & & 0.0013 \\
\hline Papillary & $725(92.0)$ & 16 & \\
\hline Other & $63(8)$ & 6 & \\
\hline Tumor size & & & $<0.0001$ \\
\hline$<4 \mathrm{~cm}$ & 555 (70.4) & 5 & \\
\hline$>4 \mathrm{~cm}$ or extrathyroid extension & $233(29.6)$ & 17 & \\
\hline Nodal information & & & $<0.0001$ \\
\hline $\mathrm{N}-$ & 375 (47.6) & 3 & \\
\hline $\mathrm{N}+$ & $413(52.4)$ & 19 & \\
\hline TNM Stage & & & $<0.0001$ \\
\hline Stage I & $585(74.2)$ & 5 & \\
\hline Stage II & $38(4.8)$ & 1 & \\
\hline Stage III & $157(19.9)$ & 15 & \\
\hline Stage IV & $8(1.0)$ & 1 & \\
\hline QTNM Stage & & & $<0.0001$ \\
\hline Low risk & $468(59.4)$ & 1 & \\
\hline Intermediate risk & 249 (31.6) & 7 & \\
\hline High risk & $71(9.0)$ & 14 & \\
\hline Treatment & & & 0.087 \\
\hline Thyroidectomy & $622(78.9)$ & 21 & \\
\hline Lobectomy & 148 (18.8) & 0 & \\
\hline Nodule excision & $6(0.8)$ & 0 & \\
\hline Not specified & $12(1.5)$ & 1 & \\
\hline
\end{tabular}

Overall 5 and 10-year survival were $98.0 \%$ and $97.0 \%$ respectively with a median follow-up of 
262.8 months (inter-quartile range, 178.8 to 389.6 months). Ten-year survival by stage are given in table 2, for QTNM and TNM. It is evident from the results in table 2 that survival is quite good and more or less similar for the low and intermediate risk groups but worse for the high risk group. In terms of TNM, 5 and 10 year survival were identical in stages I and II while stage III-IV of TNM and the high risk group of QTNM showed considerable overlap. There were however insufficient numbers within TNM stage IV to characterise it accurately.

Table 2. 5 year and 10 year cancer specific survival by TNM and QTNM stage for differentiated thyroid cancer

TNM Stage

Stage I

Stage II

Stage III

Stage IV

QTNM Stage

1 (low risk)

2 (intermediate risk)

3 (high risk)

\section{5 year CSS $^{1} \quad 10$ year CSS $^{1}$}

$\begin{array}{ll}99.3 \%(98.2-99.7) & 99.0 \%(97.4-99.6) \\ 100 \% & 100 \% \\ 92.9 \%(87.5-96.0) & 88.7 \%(81.7-93.2) \\ 87.5 \%(38.7-98.1) & 87.5 \%(38.7-98.1) \\ & \\ 100 \% & 99.6 \%(96.9-99.9) \\ 97.6 \%(94.7-98.9) & 97.0 \%(93.8-98.6) \\ 85.4 \%(74.6-91.9) & 78.6 \%(65.4-87.3)\end{array}$

${ }^{1} \mathrm{CSS}=$ cancer specific survival.

Cox proportional hazards regression of the staging systems confirms the survival findings. In particular, TNM stages I and II are statistically identical. The Kaplan-Meier plots in figure 1A and 1B for TNM and QTNM staging systems depict the increasing hazard ratio by stage reported in table 3. Again it is evident that TNM stages I-II and III-IV were comparable to QTNM low and high risk groups. Royston's D was 0.698 for QTNM and 0.473 for TNM suggesting better separation between survival distributions under the proportional hazards assumption for QTNM. 
Table 3. Hazard ratio (HR) for cancer specific death by TNM and QTNM stage

$\begin{array}{lccc}\text { Classification } & \text { Number of patients } & \begin{array}{c}\mathbf{H R}^{\mathbf{1}} \\ (\mathbf{9 5 \%} \mathbf{C I})\end{array} & \boldsymbol{P} \text { value } \\ & & & \\ \text { TNM stage } & & & \\ & & & \\ \text { Stage I } & 585 & 3.5(0.4-29.8) & 0.256 \\ \text { Stage II } & 38 & 12.9(4.7-35.5) & <0.001 \\ \text { Stage III } & 157 & 19.2(2.2-165.0) & 0.007 \\ \text { Stage IV } & 8 & & \\ \text { QTNM stage } & & & \\ \text { 1 (score 0-5; low risk) } & 468 & 1(\mathrm{ref}) & \\ \text { 2 (score 6-10; intermediate risk) } & 249 & 14.2(1.7-115.6) & 0.013 \\ \text { 3 (score 11-15; high risk) } & 71 & 117.9(15.4-900.5) & <0.001\end{array}$

${ }^{1}$ Hazard ratio interpreted as the odds that an individual in the group with the higher risk will reach the endpoint (cancer specific death) first according to the various classification systems via multivariable Cox regression analysis. Royston's D was 0.698 for QTNM and 0.473 for TNM suggesting better separation between survival distributions under the proportional hazards assumption for QTNM

This is also supported by the cross-tabulation of subjects according to TNM and QTNM depicted in table 4; there is a reasonable redistribution of patients from TNM stage I to QTNM intermediate risk and TNM Stage III to QTNM high risk, indicating that QTNM brings different prognostic value to TNM. Harrell's C statistic with a value of 0.878 for QTNM and 0.784 for TNM suggested that the proportion of correct predictions according to prognostic stage was better for QTNM than for TNM (Table 4).

However, both prognostic systems had C statistics that suggested good discrimination of survival by stage.

Finally, survival analysis was done for the 2072 patients that could be staged by TNM-7 and results are given in supplementary table S1 and figure S1. Results by stage did not differ appreciably from the cohort of 788 patients analysed above.

Table 4 - Cross tabulation of subjects by QTNM and TNM staging systems. Harrell's C was 0,878 for QTNM and 0.784 for TNM suggesting that QTNM had better discrimination of survival by stage

\begin{tabular}{lcccc} 
& \multicolumn{4}{c}{ TNM Stage } \\
QTNM Staging & Stage I & Stage II & Stage III & Stage IV \\
Low risk & 438 & 26 & 4 & 0 \\
Intermediate risk & 145 & 11 & 92 & 1 \\
High risk & 2 & 1 & 61 & 7
\end{tabular}




\section{Discussion}

This study confirms that despite the simplicity of QTNM, there is no loss of prognostic information when compared to the TNM staging system. This also suggests that prognostic stratification is not an exact science, and therefore the increase in detail obtained from binning does not translate to a better discriminative outcome. Therefore, the fine-tuning of the system, for example the splitting of T1 into T1a (for tumours $\leq 1 \mathrm{~cm}$ limited to the thyroid) and T1b (for tumours $>1 \mathrm{~cm}$ and $\leq 2 \mathrm{~cm}$ limited to the thyroid) in the latest revision of TNM [9], cannot be expected to result in a clear-cut survival benefit of a specific amount. The effect of prognostic variables will influence clinical outcome on average and it is hardly likely that there will be a specific survival gain associated with more precise classifications, as the TNM system attempts to achieve with a system of bins. In this situation, we advocate that prognostic variables be directly associated with stage through a quantitative system rather than through the complex system of binning as occurs with TNM. There are three other key benefits to this quantitative scheme: (a) as new non-anatomic prognostic variables emerge, they can easily be incorporated into quantitative systems, (b) it allows for much easier implementation in clinical practice, and (c) only variables with clear prognostic implications will be included. The potential future variables, such as BRAF mutations [32,33], tumour grading [34,35], DNA ploidy [36], nuclear atypia [37], gross lymph node involvement [38], microscopic tumour multifocality [12], macroscopic tumour multifocality [12,39], histologic PTC variants [40], size of lymph node metastases [41], and tumour angioinvasion [42] can be investigated further but must only be incorporated if they provide additional prognostic value and if consensus has been obtained regarding their use.

All prognostic stratification schemes for DTC use similar prognostic variables and three of the four prognostic factors used in QTNM were originally selected based on a multivariate analysis using a Cox proportional hazards model. The latter authors reported then that the most significant prognostic variable was tumour size (over $4 \mathrm{~cm}$ or extracapsular invasion) [10]. While there are some conflicting 
reports [43,13], this is consistent with the current literature, as size of the tumour has been strongly associated with prognosis [20] although cut-off values that have been suggested have varied and range from $1.5 \mathrm{~cm}-5 \mathrm{~cm}[15,44,35,45,46,12,39,47]$. Extrathyroidal invasion is also well known as a prognostic marker in a number of studies $[48,47,45,49,43,13]$, and thus the inclusion of both in the QTNM would be expected to improve prognostic discrimination. This is also consistent with the T parameter grouping used within the TNM [9].

The next most important prognostic variables in the QTNM were age and node status and both are supported by the existing literature. Several studies report that age is a strong predictor of poor prognosis $[4,48,49]$, and the evidence from these studies suggest an age cut-off for worse prognosis above an age of about 40 years $[45,27,47,24]$, or above age 45 years $[43,39,44,46,12,14]$, and in some reports even above age 50-55 years [50,13,15,47,24]. QTNM uses age 45 as a cut-off value, which is consistent with this literature. The other variable, lymph node metastases, was shown in the original study to have a stronger relationship with recurrence than death, although an association exists with both. Again, the literature supports these findings $[20,13,51]$ although certain studies show that the location of nodal involvement is more significant than the involvement itself [52] and yet others suggest its prognostic value is still unclear $[53,54,43,55]$.

The least influential variable in QTNM was histopathology. It was forced into the original model due to the presence of reasonable evidence, in the literature, for its status as a prognostic variable $[20,55]$, as suggested by the fact that there is a higher overall mortality in patients with FTC compared with PTC [55,4]. One likely explanation [10] is that FTC as compared to PTC presents with a higher stage and this probably suggests an underlying difference in aggressiveness.

Two variables that were not included in QTNM are gender and distant metastases at diagnosis. While DTC has a female preponderance [4], it has not been found to have any prognostic significance in many studies of DTC $[10,56,57,43,20,39]$. However, distant metastases at diagnosis is of clear prognostic significance $[55,58,43,24,20,39]$ and indeed is included as part of a number of staging systems 
$[27,9,48,12,47,15]$. The exclusion of this variable from QTNM is justified because, it plays very little role in determination of treatment, given that all patients with distant metastases are mandatorily required to be treated aggressively post-thyroidectomy. Also, being relatively uncommon, it may have less impact on risk stratification in staging systems and, additionally, its prognostic significance may in fact be modulated by age since it has been recognised that it has less effect on long-term survival in subjects below the age of 45 years [59,9]. The deliberate exclusion of metastases has been considered to increase the prognostic significance of the existing variables while making QTNM more clinically relevant [60].

It is clear from the preceding discussion that while a myriad of staging systems exist, (a) they all use the same or similar prognostic variables and (b) TNM is still considered the most discriminative of all these systems. Given the current evidence, there is little justification for the lack of a consensus on an optimal staging system. We believe that the TNM system with its associated variables currently represents the most discriminative staging system and the variant of the TNM that is represented by the QTNM makes it more clinically relevant and upgradeable in the future. The other staging systems such as MACIS [27], EORTC [48], NTCTCS [12], AMES [47], UA-MDCC [15] and others do not substantially lead to an increase in discriminative performance and their persistence hinges on institutional affiliation, as centres that proposed them continue to use them.

Despite the TNM staging system being most discriminative, it has long been known that there is overlap between its lower stages. For example, Hundahl et al [4] found that it failed to discriminate between patients with Stage I and II disease at 5 years, and Tran Cao et al [11] found that in patients over 45, there was no significant increase in risk between stage I and II. Sherman et al [12] also found that stage I and II were indistinguishable and Cady [61], upon reviewing the latter study, suggests that it is artificial to separate stages I and II. We agree with Cady, in implicitly recognizing the need for only a three level staging system and we advocate this as the optimal number. Additionally we demonstrate (Table 4) that a substantial number of TNM stage I/II patients get reclassified as intermediate risk under the QTNM system. Also, , despite it's three stages, the QTNM (based on hazard ratios) has more 
discriminatory power when comparing intermediate and high risk groups than comparing low and intermediate risk groups. This trend was shown both in the original paper and in this current validation using our data. It should be kept in mind however that the QTNM is based on DFS and it is in that scenario that discrimination into three risk strata is optimal. Prolonged survival is possible, even after disease recurrence, and it is likely that while three risk categories are optimal for DFS, in terms of CSS, there may be just a low-to-intermediate and high risk group. However, given the discrimination between low and intermediate risk in this study, it would be prudent to keep the staging system consistent for DFS and CSS.

A limitation of this study is the absence of recurrence data in our database and thus disease-free survival could not be evaluated. Although QTNM was originally developed based on recurrence [10], it also provides prognostic information about cancer specific survival and this is what we have addressed in this paper. Another limitation of this study is the absence of data on the extent of surgery and postsurgical remnant ablation with radioactive iodine. However, all patients were treated mainly at one major center in Brisbane, and the approach to therapy, up to two years ago had been total thyroidectomy followed by radioactive iodine ablation if the thyroid tumor size was greater than $1.5 \mathrm{~cm}$ in diameter. Even if there were modifications to protocol based on individual patient needs, there would be an expectation of more aggressive therapy for high risk subjects and therefore any bias introduced by treatment would only be expected to result in a decrease in discrimination in the model.

\section{Conclusion}

In conclusion, the QTNM is a simple and effective method of prognostic stratification for both recurrence and cancer specific survival, and is an adequate reflection of our current understanding of thyroid cancer [60]. There is no loss of discrimination when compared to current systems, including its parent TNM staging system, despite being simple and less complicated. Based on the fact that now the QTNM results have been confirmed in three separate patient groups across three geographical regions, we 
recommend the replacement of the standard TNM system with the QTNM system.

Declaration of interests: none 


\section{References}

1. Bray, F., Ren, J.S., Masuyer, E., Ferlay, J.: Global estimates of cancer prevalence for 27 sites in the adult population in 2008. International Journal of Cancer (2012). doi:10.1002/ijc.27711

2. Ferlay J, S.H., Bray F, Forman D, Mathers C and Parkin DM: GLOBOCAN 2008 v2.0, Cancer Incidence and Mortality Worldwide: IARC CancerBase No. 10 [Internet]. http://globocan.iarc.fr, (2010).

3. Australian Institute of Health and Welfare (AIHW) Canberra, 2012. ACIM (Australian Cancer Incidence and Mortality) Books. AIHW

4. Hundahl, S.A., Fleming, I.D., Fremgen, A.M., Menck, H.R.: A National Cancer Data Base report on 53,856 cases of thyroid carcinoma treated in the U.S., 1985-1995. Cancer 83(12), 2638-2648 (1998). doi:10.1002/(sici)1097-0142(19981215)83:12<2638::aid-cncr31>3.0.co;2-1

5. Cooper, D.S., Doherty, G.M., Haugen, B.R., Kloos, R.T., Lee, S.L., Mandel, S.J., Mazzaferri, E.L., McIver, B., Pacini, F., Schlumberger, M., Sherman, S.I., Steward, D.L., Tuttle, R.M.: Revised American Thyroid Association management guidelines for patients with thyroid nodules and differentiated thyroid cancer. Thyroid : official journal of the American Thyroid Association 19(11), 1167-1214 (2009). doi:10.1089/thy.2009.0110

6. Burke, H.B., Henson, D.E.: Criteria for prognostic factors and for an enhanced prognostic system. Cancer 72(10), 3131-3135 (1993). doi:10.1002/1097-0142(19931115)72:10<3131::aidcncr2820721039>3.0.co;2-j

7. Burke, H.B.: Outcome Prediction and the Future of the TNM Staging System. Journal of the National Cancer Institute 96(19), 1408-1409 (2004). doi:10.1093/jnci/djh293 
8. Hermanek, P., Sobin, L.H., Wittekind, C.: How to improve the present TNM staging system. Cancer 86(11), 2189-2191 (1999). doi:10.1002/(sici)1097-0142(19991201)86:11<2189::aidcncr1>3.0.co;2-r

9. Egner, J.R.: AJCC Cancer Staging Manual, Seventh ed. Springer, New York (2010)

10. Onitilo, A.A., Engel, J.M., Lundgren, C.I., Hall, P., Thalib, L., Doi, S.A.R.: Simplifying the TNM System for Clinical Use in Differentiated Thyroid Cancer. Journal of Clinical Oncology 27(11), 1872-1878 (2009). doi:10.1200/jco.2008.20.2382

11. Tran Cao, H.S., Johnston, L.E., Chang, D.C., Bouvet, M.: A critical analysis of the American Joint Committee on Cancer (AJCC) staging system for differentiated thyroid carcinoma in young patients on the basis of the Surveillance, Epidemiology, and End Results (SEER) registry. Surgery 152(2), 145-151 (2012). doi:10.1016/j.surg.2012.02.015

12. Sherman, S.I., Brierley, J.D., Sperling, M., Ain, K.B., Bigos, S.T., Cooper, D.S., Haugen, B.R., Ho, M., Klein, I., Ladenson, P.W., Robbins, J., Ross, D.S., Specker, B., Taylor, T., Maxon, H.R.: Prospective multicenter study of thyroid carcinoma treatment. Cancer 83(5), 1012-1021 (1998). doi:10.1002/(sici)1097-0142(19980901)83:5<1012::aid-cncr28>3.0.co;2-9

13. Ito, Y., Miyauchi, A., Jikuzono, T., Higashiyama, T., Takamura, Y., Miya, A., Kobayashi, K., Matsuzuka, F., Ichihara, K., Kuma, K.: Risk Factors Contributing to a Poor Prognosis of Papillary Thyroid Carcinoma: Validity of UICC/AJCC TNM Classification and Stage Grouping. World J. Surg. 31(4), 838-848 (2007). doi:10.1007/s00268-006-0455-0

14. Jukkola, A., Bloigu, R., Ebeling, T., Salmela, P., Blanco, G.: Prognostic factors in differentiated thyroid carcinomas and their implications for current staging classifications. Endocrine-Related Cancer 11(3), 571-579 (2004). doi:10.1677/erc.1.00826 
15. Beenken, S., Roye, D., Weiss, H., Sellers, M., Urist, M., Diethelm, A., Goepfert, H.: Extent of surgery for intermediate-risk well-differentiated thyroid cancer. The American Journal of Surgery 179(1), 51-56 (2000). doi:10.1016/s0002-9610(99)00254-8

16. Kahn, C., Simonella, L., Sywak, M., Boyages, S., Ung, O., O'Connell, D.: Postsurgical pathology reporting of thyroid cancer in New South Wales, Australia. Thyroid : official journal of the American Thyroid Association 22(6), 604-610 (2012). doi:10.1089/thy.2011.0501

17. Harrell, F.E., Jr., Lee, K.L., Mark, D.B.: Multivariable prognostic models: issues in developing models, evaluating assumptions and adequacy, and measuring and reducing errors. Statistics in medicine 15(4), 361-387 (1996). doi:10.1002/(sici)1097-0258(19960229)15:4<361::aid$\operatorname{sim} 168>3.0 . \operatorname{co} ; 2-4$

18. Royston, P., Sauerbrei, W.: A new measure of prognostic separation in survival data. Statistics in medicine 23(5), 723-748 (2004). doi:10.1002/sim.1621

19. Brierley, J.D., Panzarella, T., Tsang, R.W., Gospodarowicz, M.K., O'Sullivan, B.: A comparison of different staging systems predictability of patient outcome. Cancer 79(12), 2414-2423 (1997). doi:10.1002/(sici)1097-0142(19970615)79:12<2414::aid-cncr18>3.0.co;2-u

20. Loh, K.-C., Greenspan, F.S., Gee, L., Miller, T.R., Yeo, P.P.B.: Pathological Tumor-Node-Metastasis (pTNM) Staging for Papillary and Follicular Thyroid Carcinomas: A Retrospective Analysis of 700 Patients. Journal of Clinical Endocrinology \& Metabolism 82(11), 3553-3562 (1997). doi:10.1210/jc.82.11.3553

21. Lang, B.H.-H., Lo, C.-Y., Chan, W.-F., Lam, K.-Y., Wan, K.-Y.: Restaging of Differentiated Thyroid Carcinoma by the Sixth Edition AJCC/UICC TNM Staging System: Stage Migration and Predictability. Ann Surg Oncol 14(5), 1551-1559 (2007). doi:10.1245/s10434-006-9242-2 
22. Lang, B.H.-H., Lo, C.-Y., Chan, W.-F., Lam, K.-Y., Wan, K.-Y.: Staging systems for papillary thyroid carcinoma: a review and comparison. Annals of surgery 245(3), 366-378 (2007). doi:10.1097/01.sla.0000250445.92336.2a

23. Verburg, F.A., Mader, U., Kruitwagen, C.L., Luster, M., Reiners, C.: A comparison of prognostic classification systems for differentiated thyroid carcinoma. Clinical Endocrinology 72(6), 830838 (2010). doi:10.1111/j.1365-2265.2009.03734.x

24. Lo, C.-Y., Chan, W.-F., Lam, K.-Y., Wan, K.-Y.: Follicular thyroid carcinoma: the role of histology and staging systems in predicting survival. Annals of surgery 242(5), 708-715 (2005).

25. Lang, B.H.-H., Lo, C.-Y., Chan, W.-F., Lam, K.-Y., Wan, K.-Y.: Staging systems for follicular thyroid carcinoma: application to 171 consecutive patients treated in a tertiary referral centre. Endocrine-Related Cancer 14(1), 29-42 (2007). doi:10.1677/erc.1.01284

26. Passler, C., Prager, G., Scheuba, C., Kaserer, K., Zettinig, G., Niederle, B.: Application of staging systems for differentiated thyroid carcinoma in an endemic goiter region with iodine substitution. Annals of surgery 237(2), 227-234 (2003). doi:10.1097/01.sla.0000048449.69472.81

27. Hay, I.D., Bergstralh, E.J., Goellner, J.R., Ebersold, J.R., Grant, C.S.: Predicting outcome in papillary thyroid carcinoma: development of a reliable prognostic scoring system in a cohort of 1779 patients surgically treated at one institution during 1940 through 1989. Surgery 114(6), 10501057; discussion 1057-1058 (1993).

28. D'Avanzo, A., Ituarte, P., Treseler, P., Kebebew, E., Wu, J., Wong, M., Duh, Q.Y., Siperstein, A.E., Clark, O.H.: Prognostic scoring systems in patients with follicular thyroid cancer: a comparison of different staging systems in predicting the patient outcome. Thyroid : official journal of the American Thyroid Association 14(6), 453-458 (2004). doi:10.1089/105072504323150778 
29. Lang, B.H.-H., Chow, S.M., Lo, C.Y., Law, S.C., Lam, K.Y.: Staging systems for papillary thyroid carcinoma: a study of 2 tertiary referral centers. Annals of surgery 246(1), 114-121 (2007). doi:10.1097/01.sla.0000262785.46403.9b

30. Shaha, A.R.: TNM Classification of Thyroid Carcinoma. World J. Surg. 31(5), 879-887 (2007). doi:10.1007/s00268-006-0864-0

31. Wada, N., Nakayama, H., Suganuma, N., Masudo, Y., Rino, Y., Masuda, M., Imada, T.: Prognostic Value of the Sixth Edition AJCC/UICC TNM Classification for Differentiated Thyroid Carcinoma with Extrathyroid Extension. Journal of Clinical Endocrinology \& Metabolism 92(1), 215-218 (2007). doi:10.1210/jc.2006-1443

32. Guerra, A., Fugazzola, L., Marotta, V., Cirillo, M., Rossi, S., Cirello, V., Forno, I., Moccia, T., Budillon, A., Vitale, M.: A High Percentage of BRAFV600E Alleles in Papillary Thyroid Carcinoma Predicts a Poorer Outcome. Journal of Clinical Endocrinology \& Metabolism 97(7), 2333-2340 (2012). doi:10.1210/jc.2011-3106

33. Marotta, V., Sapio, M.R., Guerra, A., Vitale, M.: BRAF mutation in cytology samples as a diagnostic tool for papillary thyroid carcinoma. Expert Opinion on Medical Diagnostics 5(4), 277-290 (2011). doi:10.1517/17530059.2011.575058

34. Hay, I.D., Grant, C.S., Taylor, W.F., McConahey, W.M.: Ipsilateral lobectomy versus bilateral lobar resection in papillary thyroid carcinoma: a retrospective analysis of surgical outcome using a novel prognostic scoring system. Surgery 102(6), 1088-1095 (1987).

35. Akslen, L.A., LiVolsi, V.A.: Prognostic significance of histologic grading compared with subclassification of papillary thyroid carcinoma. Cancer 88(8), 1902-1908 (2000). doi:10.1002/(sici)1097-0142(20000415)88:8<1902::aid-cncr20>3.0.co;2-y 
36. Pasieka, J.L., Zedenius, J., Auer, G., Grimelius, L., Hoog, A., Lundell, G., Wallin, G., Backdahl, M.: Addition of nuclear DNA content to the AMES risk-group classification for papillary thyroid cancer. Surgery 112(6), 1154-1159; discussion 1159-1160 (1992).

37. Akslen, L.A.: Prognostic importance of histologic grading in papillary thyroid carcinoma. Cancer 72(9), 2680-2685 (1993). doi:10.1002/1097-0142(19931101)72:9<2680::aidcncr2820720926>3.0.co;2-d

38. Noguchi, S., Murakami, N., Kawamoto, H.: Classification of papillary cancer of the thyroid based on prognosis. World J. Surg. 18(4), 552-557 (1994). doi:10.1007/bf00353763

39. Konturek, A., Barczynski, M., Nowak, W., Richter, P.: Prognostic factors in differentiated thyroid cancer--a 20-year surgical outcome study. Langenbeck's Archives of Surgery 397(5), 809-815 (2012). doi:10.1007/s00423-011-0899-z

40. Sebastian, S., Gonzalez, J., Paricio, P., Perez, J., Flores, D., Madrona, A., Romero, P., Tebar, F.: Papillary thyroid carcinoma: Prognostic index for survival including the histological variety. Archives of Surgery 135(3), 272-277 (2000). doi:10-1001/pubs.Arch Surg.-ISSN-0004-0010135-3-soa9035

41. Sugitani, I., Kasai, N., Fujimoto, Y., Yanagisawa, A.: A novel classification system for patients with PTC: addition of the new variables of large $(3 \mathrm{~cm}$ or greater) nodal metastases and reclassification during the follow-up period. Surgery 135(2), 139-148 (2004). doi:10.1016/s00396060(03)00384-2

42. Yildirim, E.: A model for predicting outcomes in patients with differentiated thyroid cancer and model performance in comparison with other classification systems. Journal of the American College of Surgeons 200(3), 378-392 (2005). doi:10.1016/j.jamcollsurg.2004.10.031 
43. Wong, R., Bresee, C., Braunstein, G.: Comparison with Published Systems of a New Staging System for Papillary and Follicular Thyroid Carcinomas. Thyroid : official journal of the American Thyroid Association (2012). doi:10.1089/thy.2012.0181

44. DeGroot, L.J., Kaplan, E.L., McCormick, M., Straus, F.H.: Natural history, treatment, and course of papillary thyroid carcinoma. The Journal of Clinical Endocrinology \& Metabolism 71(2), 414424 (1990).

45. Mazzaferri, E.L., Jhiang, S.M.: Long-term impact of initial surgical and medical therapy on papillary and follicular thyroid cancer. The American Journal of Medicine 97(5), 418-428 (1994). doi:10.1016/0002-9343(94)90321-2

46. Shaha, A.R., Loree, T.R., Shah, J.P.: Intermediate-risk group for differentiated carcinoma of thyroid. Surgery 116(6), 1036-1040; discussion 1040-1031 (1994).

47. Cady, B., Rossi, R.: An expanded view of risk-group definition in differentiated thyroid carcinoma. Surgery 104(6), 947-953 (1988).

48. Byar, D.P., Green, S.B., Dor, P., Williams, E.D., Colon, J., van Gilse, H.A., Mayer, M., Sylvester, R.J., Van Glabbeke, M.: A prognostic index for thyroid carcinoma. A study of the E.O.R.T.C. thyroid cancer cooperative group. European Journal of Cancer 15(8), 1033-1041 (1979). doi:10.1016/0014-2964(79)90291-3

49. Lerch, H., Schober, O., Kuwert, T., Saur, H.B.: Survival of differentiated thyroid carcinoma studied in 500 patients. Journal of Clinical Oncology 15(5), 2067-2075 (1997).

50. Ito, Y., Ichihara, K., Masuoka, H., Fukushima, M., Inoue, H., Kihara, M., Tomoda, C., Higashiyama, T., Takamura, Y., Kobayashi, K., Miya, A., Miyauchi, A.: Establishment of an Intraoperative 
Staging System (iStage) by Improving UICC TNM Classification System for Papillary Thyroid Carcinoma. World J. Surg. 34(11), 2570-2580 (2010). doi:10.1007/s00268-010-0710-2

51. Sellers, M., Beenken, S., Blankenship, A., Soong, S.-j., Turbat-Herrera, E., Urist, M., Maddox, W.: Prognostic significance of cervical lymph node metastases in differentiated thyroid cancer. The American Journal of Surgery 164(6), 578-581 (1992). doi:10.1016/s0002-9610(05)80710-x

52. de Meer, S.G., Dauwan, M., de Keizer, B., Valk, G.D., Borel Rinkes, I.H., Vriens, M.R.: Not the number but the location of lymph nodes matters for recurrence rate and disease-free survival in patients with differentiated thyroid cancer. World J. Surg. 36(6), 1262-1267 (2012).

53. Sato, N., Oyamatsu, M., Koyama, Y., Emura, I., Tamiya, Y., Hatakeyama, K.: Do the level of nodal disease according to the TNM classification and the number of involved cervical nodes reflect prognosis in patients with differentiated carcinoma of the thyroid gland? Journal of Surgical Oncology 69(3), 151-155 (1998). doi:10.1002/(sici)1096-9098(199811)69:3<151::aidjso6>3.0.co;2-v

54. Beasley, N., Lee, J., Eski, S., Walfish, P., Witterick, I., L., F.J.: Impact of nodal metastases on prognosis in patients with well-differentiated thyroid cancer. Archives of Otolaryngology-Head \& Neck Surgery 128(7), 825-828 (2002). doi:10.1001/archotol.128.7.825

55. Lundgren, C.I., Hall, P., Dickman, P.W., Zedenius, J.: Clinically significant prognostic factors for differentiated thyroid carcinoma. Cancer 106(3), 524-531 (2006). doi:10.1002/cncr.21653

56. Shaha, A.: Treatment of thyroid cancer based on risk groups. Journal of Surgical Oncology 94(8), 683-691 (2006). doi:10.1002/jso.20697 
57. Pelizzo, M.R., Boschin, I.M., Toniato, A., Piotto, A., Pagetta, C., Gross, M.D., Al-Nahhas, A., Rubello, D.: Papillary thyroid carcinoma: 35-year outcome and prognostic factors in 1858 patients. Clinical nuclear medicine 32(6), 440-444 (2007). doi:10.1097/RLU.0b013e31805375ca

58. Haq, M., Harmer, C.: Differentiated thyroid carcinoma with distant metastases at presentation: prognostic factors and outcome. Clinical Endocrinology 63(1), 87-93 (2005). doi:10.1111/j.13652265.2005.02304.x

59. Clark, J.R., Lai, P., Hall, F., Borglund, A., Eski, S., Freeman, J.L.: Variables predicting distant metastases in thyroid cancer. The Laryngoscope 115(4), 661-667 (2005).

60. Lang, B.H.-H., Lo, C.-Y.: Cancer: QTNM: a simplified TNM or just another staging system? Nature Reviews Endocrinology 5(10), 531-532 (2009).

61. Cady, B.: Staging in thyroid carcinoma. Cancer 83(5), 844-847 (1998). 